\title{
Physics' Need for Interval Uncertainty and How It Explains Why Physical Space Is (at Least) 3-Dimensional
}

\author{
Olga Kosheleva and Vladik Kreinovich \\ University of Texas at El Paso \\ $500 \mathrm{~W}$. University \\ El Paso, TX 79968, USA \\ olgak@utep.edu,vladik@utep.edu
}

\begin{abstract}
One of the fundamental problems of modern physics is the problem of divergence: e.g., when we try to compute the overall energy of the electric field generated by a charged elementary particle, we get a physically meaningless infinite value. In this paper, we show that one way to avoid these infinities is to take into account that measurements are always imprecise - and thus, we never get the exact values of the physical quantities, only intervals of possible values. We also show that 3-dimensional space is the simplest one in which such interval uncertainty is inevitable. This may explain why the physical space is (at least) 3-dimensional.
\end{abstract}

Keywords: divergence problem in physics, interval uncertainty, fuzzy uncertainty, physical dimension

\section{Divergence Problem in Physics}

Divergence problem: a brief reminder. The known divergence problem (see, e.g., $[5,14]$ ) is related to the following simple question: what is the mass of an electron? More generally, what is mass of a charged elementary particle - or, even more generally, the mass of any elementary particle which is involved in interactions of some type?

In general, this mass $m$ consists of two parts:

- the mass $m_{0}$ of the particle itself, and

- the mass $m_{f}=\frac{E_{f}}{c^{2}}$ which is equivalent to the total energy $E_{f}$ of the electric (or other) field generated by the particle:

$$
m=m_{0}+m_{f} .
$$

According to special relativity, an elementary particle, i.e., a particle that cannot be further subdivided, is a point-wise object - otherwise, if it was not pointwise, different spatial locations within the same particle would be, in general, different, since they cannot instantaneously interact. For a point-wise particle, the electric field at a distance $r$ from the particle is proportional to $r^{-2}$ :

$$
E=c_{E} \cdot r^{-2} .
$$

The energy density $\rho$ of the field is proportional to $E^{2}$, thus, $\rho=c_{\rho} \cdot r^{-4}$, for an appropriate constant $c_{\rho}$. The overall energy $E_{f}$ of the electric field can be obtained by integrating $\rho(x)$ over the whole 3 -D space:

$$
\begin{gathered}
E_{f}=\int \rho(x) d^{3} x=\int c_{\rho} \cdot r^{-4} d^{3} x= \\
\int_{r=0}^{r=\infty} c_{\rho} \cdot r^{-4} \cdot 4 \pi \cdot r^{2} d r .
\end{gathered}
$$

Thus,

$$
E_{f}=c_{f} \cdot \int_{0}^{\infty} \frac{d r}{r^{2}}=\left.c_{f} \cdot \frac{1}{r}\right|_{r=0} ^{r=\infty},
$$

where $c_{f} \stackrel{\text { def }}{=} c_{\rho} \cdot 4 \pi$.

At $r=0$, the expression $\frac{1}{r}$ tends to infinity, so we conclude that $E_{f}=\infty$ and thus, that the overall mass $m=m_{0}+\frac{E_{f}}{c^{2}}$ of the electron is ... infinite.

Divergence is caused only by scale-invariance. The divergence problem does not disappear if we change the formula for the dependence of the field on distance and/or change the dimension - as long as we keep the formulas scale-invariant in the following sense.

The standard formulas for electromagnetic interactions (and for many other interactions) do not have a preferred unit of length. As a result, formulas should not change if we simply change the unit of length - 
e.g., replace meters with feet. If we replace the original unit with a unit which is $\lambda$ times smaller, then all the numerical values of distance are multiplied by $\lambda$, i.e., each distance $r$ in the old units becomes $r^{\prime} \stackrel{\text { def }}{=} \lambda \cdot r$ in the new units.

Of course, the dependence $y(r)$ of a relevant physical quantity $y$ (the field itself or the field's energy density) on the distance $r$ cannot be literally the same: otherwise, from the condition that $y(\lambda \cdot r)=y(r)$ we should be able to conclude that $y(r)=$ const. The reason for this impossibility is that measuring units for different quantities are often related: e.g., when we change units for distance $d$, to preserve the formulas like $d=v \cdot t$, where $v$ denotes velocity and $t$ denotes times, we should accordingly change the unit for velocity $v$.

In our case, for each $\lambda$, we not only replace $x$ with $x^{\prime}=\lambda \cdot x$, we also replace the numerical $y$ with the appropriately re-scaled value $y^{\prime}=c(\lambda) \cdot y$. We can now require that when $y=f(x)$, then $y^{\prime}=f\left(x^{\prime}\right)$. Substituting the expressions for $x^{\prime}$ and $y^{\prime}$ into this formula, we conclude that $f(\lambda \cdot x)=c(\lambda) \cdot f(x)$. For continuous (even for measurable) functions $f(x)$ this equality implies that $f(x)=A \cdot x^{c}$ for some real numbers $A$ and $c$; see, e.g., [1]. In particular, scale-invariance implies the power law dependence of the energy density $\rho(r)$ on the distance $r$ from the particle: $\rho(r)=A \cdot r^{c}$.

In an $m$-dimensional space, for a spherically symmetric function $\rho(r)$ (i.e., a function depending only on $r$ ), we thus have

$$
\begin{gathered}
E_{f}=\int \rho(r) d^{m} x=A \cdot \int r^{c} d^{m} x= \\
A \cdot \text { const } \cdot \int_{0}^{\infty} r^{c} \cdot r^{m-1} d r= \\
\text { const } \cdot \int_{0}^{\infty} r^{c+m-1} d r=\text { const }\left.\cdot \frac{r^{c+m}}{c+m}\right|_{0} ^{\infty} .
\end{gathered}
$$

This expression is always infinite:

- when $c+m>0$, it is infinite for $r=\infty$;

- when $c+m<0$, the above expression is infinite for $r=0$;

- finally, when $c+m=0$, the integral of $r^{c+m-1}=$ $r^{-1}$ is equal to $\ln (r)$, and is, therefore, infinite both at $r=0$ and at $r=\infty$.

Thus, the divergence problem indeed follows from scale-invariance, and it does not depend on what exactly is the dependence on the field on the distance or on what is the dimension of the physical space.
How the divergence problem is solved now. The usual solution to the divergence problem is renormalization. Crudely speaking, we take $m_{0}=-\infty$, so that the sum of minus infinity $m_{0}$ and plus infinity $m_{f}=\frac{E_{f}}{c^{2}}$ is finite. To implement this idea, instead of considering point-wise particles, we consider particles of a finite radius $\varepsilon$. In this case, the overall energy $E_{f}(\varepsilon)$ is finite. We then take $m_{0}(\varepsilon)=m-\frac{E_{f}(\varepsilon)}{c^{2}}$, where $m$ is the empirically observed electron's mass. In the limit $\varepsilon \rightarrow 0$, the proper mass $m_{0}(\varepsilon)$ tends to $-\infty$, the energy $E_{f}(\varepsilon)$ tends to $+\infty$, but their sum remains equal to the same constant $m$.

Problem with renormalization. From the mathematical viewpoint, renormalization solves the divergence problem. However, from the physical viewpoint, renormalization looks like a trick.

Alternative approaches: main idea and limitations. Since the existing physical equations - while being well-confirmed by experiments - lead to physically meaningless infinities, a natural idea is to modify these equations so as to avoid these infinities. Such modifications have indeed been proposed, to avoid both $r=0$ and $r=\infty$ infinities.

To avoid infinities at $r=\infty$, a physically reasonable idea is to take into account that many physical interactions - such as strong ones - correspond to exchanging quanta of finite rest mass. For such interactions, the force exponentially decreases with distance, and this decrease makes the corresponding integrals finite. So, all $r=\infty$ problems will disappear if we assume that, e.g., photons - quanta of electromagnetic interactions - also have a non-zero rest mass.

To avoid infinities at $r=0$, a natural idea is to modify the formulas corresponding to small $r$. This can be done, e.g., by considering physical theories in which there is a quantum of length ("elementary length") - or, more generally, in which there is a lower limit on the size of the regions that we can measure; see, e.g., $[6]$.

The main problem with these approaches is that for the physicists to adopt a new alternative physical theory, this theory must be experimentally confirmed. And so far, there has been no experimental data confirming any of the proposed new alternative theories - all the available evidence seems to support the existing theories. Thus, from the physical viewpoint, these alternative approaches also sounds like mathematical tricks designed to avoid a mathematical problem - rather than a convincing physical theory.

Remaining challenge. It is therefore desirable to come up with a more physically meaningful way to 
avoid infinities. This is what we try to do in this paper.

\section{Physics' Need for Interval Uncertainty}

Main idea: interval uncertainty. In explaining the divergence problem, we implicitly assumed that each physical quantity can be, in principle, measured with any possible accuracy - and thus, that each quantity can be characterized by an exact number.

In practice, measurements also have some imprecision, and it is reasonable to consider the case when we cannot perform measurements beyond a certain level of accuracy. In this case, we will never know the exact value of the corresponding quantity; we will only know the interval containing this quantity.

Let us see how this possibility affects the divergence.

\section{Comments.}

- Our analysis of the effect of interval uncertainty on divergence expands the idea presented in [8].

- At first glance, our idea may sound similar to elementary-length theories. However, the difference is that, in contrast to these theories, we do not change the original theory, we do not assume any universally valid lower bound on distances or accuracies - we are simply using the well-known fact that each measurement has a finite accuracy.

Interval uncertainty helps avoid divergence. Let us assume the distance $r$ can only be measured with accuracy $r_{0}$ (and is, thus, defined only with this accuracy). In this case, when the measured distance is $r$, the actual distance can take all possible values from $r-r_{0}$ (to be more precise, from $\max \left(r-r_{0}, 0\right)$ since the distance is always non-negative) to $r+r_{0}$.

The expression $\rho(x)=c_{\rho} \cdot r^{-4}$ for the dependence of energy density $\rho$ on the distance $r$ is decreasing. Thus, the density $\rho(x)$ of the energy field can take any value from $\rho(x)=c_{\rho} \cdot\left(r+r_{0}\right)^{-4}$ to

$$
\bar{\rho}(x)=c_{\rho} \cdot\left(\max \left(0, r-r_{0}\right)\right)^{-4} .
$$

Therefore, the total energy $E_{f}$ of the particle's electric field lies between the values

$\underline{E}_{f}=\int c_{\rho} \cdot\left(r+r_{0}\right)^{-4} d^{3} x=4 \pi \cdot c_{\rho} \cdot \int_{0}^{\infty} r^{2} \cdot\left(r+r_{0}\right)^{-4} d r$

and

$$
\begin{aligned}
& \bar{E}_{f}=\int c_{\rho} \cdot\left(\max \left(0, r-r_{0}\right)\right)^{-4} d^{3} x= \\
& 4 \pi \cdot c_{\rho} \cdot \int_{0}^{\infty} r^{2} \cdot\left(\max \left(0, r-r_{0}\right)\right)^{-4} d r .
\end{aligned}
$$

The upper bound $\bar{E}_{f}$ is infinite, but the lower bound $\underline{E}_{f}$ is finite, since the lower density is bounded by a constant in the vicinity of $r=0$. Thus, the fact that we have an infinite upper bound does not imply that the energy is infinite: it can be finite, as long as it is larger than or equal to $\underline{E}_{f}$.

In short, in the presence of interval uncertainty, there is no divergence.

\section{Comments.}

- It is easy to see that the divergence at $r=0$ disappears for all possible power law dependencies $\rho(x) \sim r^{c}$ and for all possible spatial dimensions.

- In addition to a fixed-accuracy interval uncertainty, we can also consider a more realistic model, in which we have different accuracies with different degree of confidence. As a result, for each quantity, instead of a single interval, we have a nested family of intervals corresponding to different degrees of confidence. This is, in effect, a fuzzy set; see, e.g., $[4,7,9,11,12,13,15]$. In this fuzzy case, we also eliminate divergence.

\section{Why Physical Space Is at Least 3-Dimensional}

Role of probabilities. According to quantum physics, all quantities can be determined only with some probabilities; see, e.g., [5, 14]. From this viewpoint, the most important quantity is the probability - i.e., in mathematical terms, a probability measure on the set of all possible events.

Probabilities can be interval-valued. Even for the usual Lebesgue measure (length, area, volume, etc.) some sets $S$ are not measurable, meaning that the lower probability $\underline{P}(S)=\sup \{P(A): A \subseteq S\}$ differs from the upper probability $\bar{P}(S)=\inf \{P(A): S \subseteq$ $A\}$. In such cases, we can say that the measure of the set $S$ is the interval $[\underline{P}(S), \bar{P}(S)]$. So, it is possible (and natural) to have interval-valued probabilities and thus, interval-type values for all other quantities, such as the expected values, moments, etc.

In 2-D case, it is possible to avoid intervals altogether. Interestingly, while interval-valued probabilities are possible in all dimensions, they are not necessarily appearing in 2-D physical space. The reason for this is that in the 2-D space, it is possible to extend Lebsgue measure to a shift- and rotationinvariant finitely-additive measure defined on all possible 2-D sets $[2,10]$. 
In contrast, in 3-D and in higher dimensions, intervals are inevitable. In 3-D space, it is known that we can decompose a unit ball (= filled sphere) into finitely many pieces, and then shift and rotate these pieces so that these shifted-and-rotated pieces form two balls identical to the original one; see, e.g., [3]. This counterintuitive possibility is known as BanachTarski paradox.

This result shows that in the 3-D case, it is not possible to extend Lebesgue to shift- and rotation-invariant finitely additive measure: otherwise, decompositions and shift would preserve overall measure, while in the context of the paradox, the final volume is twice larger that the original one. A similar construction is possible for all higher dimensions.

This explains why the physical space is at least 3-dimensional. As we have mentioned earlier, to avoid physically meaningless divergences, it is necessary to have interval uncertainty. In $1-\mathrm{D}$ and $2-\mathrm{D}$ cases, it is possible to avoid interval uncertainty and thus, get divergence. Starting with dimension 3 , however, interval values are inevitable and thus, divergence is not possible. This explains why the physical space is at least 3-dimensional - with the usual 3-D physical space being the simplest space with this property.

\section{Acknowledgments}

This work was partially supported by the US National Science Foundation via grant HRD-1242122 (CyberShARE Center of Excellence).

The authors are thankful to the anonymous referees for valuable suggestions.

\section{References}

[1] J. Aczél and J. Dhombres, Functional Equations in Several Variables, Cambridge University Press, 2008.

[2] S. Banach, "Sur le problème de la mésure", Fundamenta Mathematicae, 1923, Vol. 4, pp. 7-33.

[3] S. Banach and A. Tarski, "Sur la décomposition des ensembles de points en parties respectivement congruentes", Fundamenta Mathematicae, 1924, Vol. 6, pp. 244-277.
[4] R. Belohlavek, J. W. Dauben, and G. J. Klir, Fuzzy Logic and Mathematics: A Historical Perspective, Oxford University Press, New York, 2017.

[5] R. Feynman, R. Leighton, and M. Sands, The Feynman Lectures on Physics, Addison Wesley, Boston, Massachusetts, 2005.

[6] S. P. Gudder, Quantum Probability, Academic Press, Dan Diego, California, 1988.

[7] G. Klir and B. Yuan, Fuzzy Sets and Fuzzy Logic, Prentice Hall, Upper Saddle River, New Jersey, 1995.

[8] O. Kosheleva and V. Kreinovich, "Interval (set) uncertainty as a possible way to avoid infinities in physical theories", Abstracts of the 18th International Symposium on Scientific Computing, Computer Arithmetic, and Verified Numerical Computation SCAN'2018, Tokyo, Japan, September 10-15, 2018.

[9] J. M. Mendel, Uncertain Rule-Based Fuzzy Systems: Introduction and New Directions, Springer, Cham, Switzerland, 2017.

[10] J. Mycielski, "Finitely additive measures. I", Colloquium Mathematicae, 1979, Vol. 42, pp. 309318.

[11] H. T. Nguyen and V. Kreinovich, "Nested intervals and sets: concepts, relations to fuzzy sets, and applications", In: R. B. Kearfott and V. Kreinovich (eds.), Applications of Interval Computations, Kluwer, Dordrecht, 1996, pp. 245290.

[12] H. T. Nguyen, C. Walker, and E. A. Walker, A First Course in Fuzzy Logic, Chapman and Hall/CRC, Boca Raton, Florida, 2019.

[13] V. Novák, I. Perfilieva, and J. Močkoř, Mathematical Principles of Fuzzy Logic, Kluwer, Boston, Dordrecht, 1999.

[14] K. S. Thorne and R. D. Blandford, Modern Classical Physics: Optics, Fluids, Plasmas, Elasticity, Relativity, and Statistical Physics, Princeton University Press, Princeton, New Jersey, 2017.

[15] L. A. Zadeh, "Fuzzy sets", Information and Control, 1965, Vol. 8, pp. 338-353. 